\title{
Evolution of Early Repolarization Patterns after 5 Years in a Military Population at Low Cardiovascular Risk and Practical Implications in Military Medical Expertise
}

\author{
Ulric Vinsonneau, M.D., ${ }^{*}$ Vanida Pangnarind-Heinz, M.D., ${ }^{*}$ \\ Nicolas Paleiron, M.D., ${ }^{*}$ Louis Marie Heinz, M.D., ${ }^{*}$ Guenole Rohel, M.D., ${ }^{*}$ \\ Marie Piquemal, M.D., ${ }^{*}$ Philippe Castellant, M.D. $\dagger \dagger$ Jerome Abalea, M.D. $\dagger$ \\ Jacques Mansourati, M.D., $\dagger$ and Philippe Paule, M.D.*
}

From the *Department of Cardiology, "Clermont Tonnerre" Hospital of Military Training, Brest, France; and $\dagger$ Department of Cardiology, "La Cavale Blanche" University Hospital Center, Brest, France Background: The French military population is a young and athletic population with a high
prevalence of early repolarization patterns (ERP) compared to the general population. Screening
of military officers at risk of sudden death (SD) is a priority in military medical expertise. The aim
of our study was to evaluate the prevalence of ERP and its evolution over a period of 5 years in a
specific asymptomatic population, free of heart disease and cardiovascular risk factors.
Methods: From March to December 2008 , we prospectively collected the electrocardiograms
(ECGs) of military officers enlisted into the submarine forces of France. For 5 years (until 2012), the
military officers included in the study underwent a clinical examination twice per year. A new ECG
was carried out 5 years after the initial one.
Results: A total of 250 male subjects were included (mean age $22.87 \pm 0.5$ years). The prevalence
of ERP was $19.2 \%$. The most common appearance was an elevation of a slurring type J point ( $31 / 48$
or $64.5 \%$ ) in inferolateral leads ( $18 / 48$ or $37.8 \%$ ). After 5 years, the prevalence of ERP was identical
to that of the previous period (19.2\%) with no change in appearance, distribution, and amplitude
in 47 subjects ( $97.9 \%$ ). Repolarization changed in two patients (one occurrence of ERP and one
disappearance). During the 5 years of follow-up, the subjects remained asymptomatic
Conclusion: ERP is common in our population of young, athletic, and asymptomatic military
officers and changes little in 5 years.

Ann Noninvasive Electrocardiol 2015;20(5):420-425

early repolarization; sudden death; young subjects

The French military population is a young, predominantly male population subject to specific physical and environmental constraints. The incidence of sudden death (SD) in young military officers under the age of 35 years is estimated at 2.5 deaths/100,000 per annum. ${ }^{1}$ One of the main missions of military cardiologists is the detection and treatment of acquired or congenital heart disease responsible for SD. To detect these heart diseases, all French military officers undergo a clinical examination and a routine electrocardiogram (ECG) during their induction and regularly throughout their career. In the presence of suggestive clinical and ECG abnormalities, the patient is addressed to the cardiology department of one of the military hospitals for further advice.

In 2008, Haissaguere ${ }^{2}$ defined early repolarization patterns (ERP) as a $\mathrm{J}$ point elevation (QRS-ST

Address for correspondence: Ulric Vinsonneau, M.D., Department of Cardiology, Clermont Tonnerre Hospital of Military Training, Brest BP41 BCRM, 29240 Brest Cedex 9, France; Fax: +33-298-437-504; E-mail: ulric.vinsonneau@orange.fr. Phone: +33-298-437-301

Funding: We did not receive any funding for this study. 


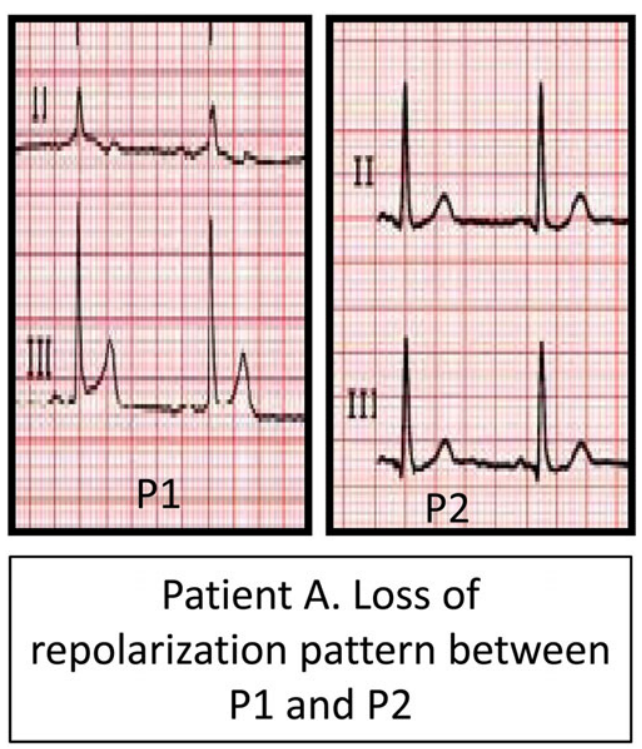

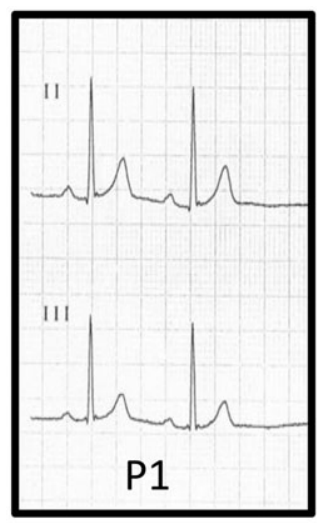

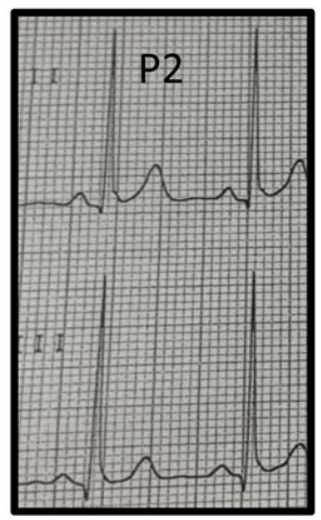

\section{Patient B. Appearance of repolarization pattern between P1 and P2}

Figure 1. Slurring and notching type appearance of early repolarization pattern.

junction), which can take the form of slurring or notching greater than or equal to $0.1 \mathrm{mV}$ in at least two lower and/or lateral leads (II, III, aVF, I, aVL, $\mathrm{V}_{4}-\mathrm{V}_{6}$ ) excluding leads $\mathrm{V}_{1}-\mathrm{V}_{3}$ and associated with a risk of SD by ventricular fibrillation (VF; Fig. 1). The screening of subjects with ERP at risk of SD is difficult because this pattern is common in the general population $(3-13 \%)$ unlike the occurrence of a VF in a healthy heart. ${ }^{2-5}$ Thus, this pattern is not sufficient in itself to identify patients at high risk of SD. Risk stratification happens in practice through investigation of clinical variables and ECG. Clinical evidence of malignancy is represented by a family history of SD before the age of 45 and unexplained syncope. ${ }^{6}$ Derogatory ECG elements combine the dynamic character and a J point amplitude greater than $0.2 \mathrm{mV}$, the appearance and distribution of a J point in the form of notching in the lower and inferolateral leads and the appearance of a horizontal or descending ST segment. ${ }^{3-8}$ The prevalence of ERP in a population of young, athletic military officers has been estimated at $13 \%$ and until 2008 its discovery did not lead to further cardiovascular explorations or restriction of employment or physical activity. ${ }^{9}$ Since then, our practical attitude comes down to a risk stratification of SD among these young military officers based on clinical variables and ECGs. The continuation of the military officer in his professional activity depends on the results of this evaluation.

\section{METHODS}

\section{Main Objective}

The main objective of the study is to estimate the prevalence, appearance, distribution, and evolution of ERP after 5 years according to Haissaguere's criteria ${ }^{2}$ in a specific population for whom we are medically liable, to optimize our decisions around fitness to work within the framework of medical expertise.

\section{Population Studied and Study Conduct}

From March to December 2008, we prospectively collected the ECGs of military officers enlisted into the submarine forces of France under the following inclusion criteria: subjects aged over 17 years, Caucasians and practicing at least two hours of sport per week. They had to be asymptomatic in cardiovascular terms (no unexplained syncope) and not have a family history of SD before the age of 45 years. They could not present cardiovascular risk factors such as hypertension (blood pressure $>140 / 90 \mathrm{mmHg}$ ), dyslipidemia (total cholesterol $>200 \mathrm{mg} / \mathrm{dL}$ ), diabetes (fasting glucose $>126 \mathrm{mg} / \mathrm{dL}$ ), active smoking, or body mass index (BMI) greater than $30 \mathrm{~kg} / \mathrm{m} .^{2}$

The exclusion criteria are as follows: all symptomatic or asymptomatic subjects with ECG abnormalities such as a complete or incomplete 
branch block, an atrioventricular block, or an atrial fibrillation were excluded from the study.

For 5 years (until 2012), the military officers included in the study underwent a clinical examination twice per year. A new ECG was carried out 5 years after the initial one. The first series of ECGs carried out in 2008 is denoted P1; the second series of ECGs carried out 5 years later (2012) is denoted P2.

\section{ECG Reading Phase}

For each period P1 and P2, the ECGs were carried out at rest, lying down, with 12 standard leads, and a rate of $25 \mathrm{~mm} / \mathrm{s}$. The ECGs were anonymous and read separately by two cardiologists in the first instance. The presence of ERP was denoted RP+, the absence of ERP was denoted RP-, and a borderline appearance denoted as "borderline." In the second instance, the ECGs were reread together. The results were validated by the agreement of three readings. In the case of "borderline" appearance, discrepancy and in the absence of consensus, the ECGs were classified as RP-. The appearance and distribution of the $\mathrm{J}$ point were specified as well as the appearance of ST elevation.

\section{Statistical Analysis}

Descriptive statistics are presented after calculation of means and percentages and determination of confidence intervals at 95\% (binomial formula). In terms of analytical statistics, the percentages were compared using the Fisher's exact test or chi-square test, after verification of performance conditions. The comparisons of means were carried out by the Student's $t$-test, after verification of performance conditions (equality of variances). The comparisons of means on paired series were also carried out using the Student's $t$-test. The level of statistical significance was set at $5 \%(\mathrm{P}<0.05)$.

\section{RESULTS}

During the study period, 250 male subjects were included. The mean age of the subjects was 22.87 \pm 0.5 years. The general characteristics of the population are summarized in Table 1. During the 5 years of follow-up, the population remained free of cardiovascular risk factors and individuals continued regular exercise $>2$ hours per week. All subjects remained asymptomatic. No SD occurred.

\section{Period P1: Analysis of ECGs}

The prevalence of RP+ was $19.2 \%$ or 48 cases. There was no statistically significant difference between the RP+ and RP- groups in terms of age. $\mathrm{RP}+$ subjects had a statistically significantly lower heart rate, a shorter QTc, and a higher Sokolow index (Table 2). The most common appearance of a J point was the slurring type (31 of 48 or $64.5 \%$ ) in the inferolateral branches (18/48 or $37.8 \%)$. The appearance of a notching type $\mathrm{J}$ point was only found in $35.5 \%$ of cases. ST elevation was ascending in $60.4 \%$ of cases. ST segment elevation greater than $2 \mathrm{~mm}$ was not found. These results are summarized in Tables 2 and 3.

\section{Period P2: Analysis of ECGs}

Five years later, the prevalence of ERP was identical to the previous period (19.2\%). RP+ subjects also had a statistically significant shorter QTc and higher Sokolow index. There was no significant difference in the HR. The predominant appearance of a J point was slurring for 32 cases $(66.7 \%)$ and inferolateral localization (19 cases or $39.5 \%)$. The ST segment elevation was less than 2 $\mathrm{mm}$ in all cases. It was ascendant in $58.3 \%$ and horizontal in $41.7 \%$. The results are summarized in Tables 2 and 3.

\section{Evolution of ERP}

There was identical persistence in terms of distribution, appearance, and amplitude of RP+ in 47 subjects (97.9\%). Heart rate is generally higher for ECG P1 than for ECG P2 without any statistically significant difference (Fig. 1). There was no significant difference between the ECG ERP+ P1 and P2 in terms of the QTc and Sokolow index. Repolarization changed in two patients. We noted the disappearance of ERP in P2 with an almost identical HR in the ECGs of P1 and P2 $\left(\mathrm{HR}_{\mathrm{P} 1}=61 \mathrm{bpm}, \mathrm{HR}_{\mathrm{P} 2}=62 \mathrm{bpm}\right)$. We found an appearance of ERP in one subject in $\mathrm{P} 2$ with a lower $\mathrm{HR}$ reading in $\mathrm{P} 1$ compared to $\mathrm{P} 2\left(\mathrm{HR}_{\mathrm{P} 1}=\right.$ 75 bpm; $\left.\mathrm{HR}_{\mathrm{P} 2}=100 \mathrm{bpm}\right)$. The appearance of a J point was inferolateral slurring associated with an ST elevation of $>1 \mathrm{~mm}$ horizontal. The results are summarized in Table 4. 
A.N.E. • September 2015 • Vol. 20, No. 5 • Vinsonneau, et al. • ERPs in the Military • 423

Table 1. General Characteristics of the Study Population

\begin{tabular}{lr}
\hline Total & $\mathbf{N}=\mathbf{2 5 0}$ \\
\hline Age (years) & $22.87 \pm 0.5$ \\
Weight (kg) & $76.7 \pm 1.5$ \\
Height (m) & $1.77 \pm 0.1$ \\
Heredity/hypertension/diabetes/dyslipidemia/smoking & 0 \\
Family history of sudden death/unexplained syncope & 0 \\
Sport practice $>$ 2 hours/week & $250(100 \%)$ \\
\hline
\end{tabular}

Table 2. Clinical and ECG Characteristics in the Periods 1 and 2 (P1-P2)

\begin{tabular}{lcccc}
\hline Population & Total, $\mathbf{n}=\mathbf{2 5 0}$ & ARP,$\pm \mathbf{n}=\mathbf{4 8}(\mathbf{1 9 . 2 \% )}$ & ARP-, $\mathbf{n}=\mathbf{2 0 2} \mathbf{( 8 0 . 8 \% )}$ & $\mathbf{P}$ \\
\hline & & Period P1 & & \\
Age & $22.87(22.4-23.35)$ & $22.65(22.3-23.4)$ & $23.7(22.9-24)$ & 0.07 \\
ECG characteristics & $68.5( \pm 12.7)$ & $66( \pm 13.5)$ & $71.7( \pm 13)$ & 0.008 \\
FC (BPM) & $388( \pm 29.2)$ & $382.7( \pm 33)$ & $394.3( \pm 29)$ & 0.008 \\
QTC Bazett (ms) & $29( \pm 0.7)$ & $31.04( \pm 0.6)$ & $27.6( \pm 1.6)$ & 0.019 \\
HVG Sokolow (mm) & Period P2 & $28.78(27.9-29)$ & 0.1 \\
Age & $27.87(27.4-28.35)$ & $27.68(27.3-28.4)$ & $67.6( \pm 13)$ & 0.03 \\
ECG characteristics & $66.8( \pm 12.7)$ & $65.8( \pm 13.5)$ & $396.4( \pm 31)$ & 0.01 \\
FC (BPM) & $392.2( \pm 30.2)$ & $388.1( \pm 33)$ & $26.97( \pm 0.7)$ & 0.019 \\
QTC Bazett (ms) & $28.5( \pm 0.6)$ & $30.4( \pm 1.2)$ & \\
HVG Sokolow (mm) & &
\end{tabular}

Table 3. Appearance-Distribution of J Point and Segment ST Elevation (P1 and P2)

\begin{tabular}{|c|c|c|c|}
\hline & $R P+P 1(n=48 / 19.2 \%)$ & $R P+P 2(n=48 / 19.2 \%)$ & $\mathbf{P}$ \\
\hline $\begin{array}{l}\text { HR (bpm) } \\
\text { QTc Bazett (ms) } \\
\text { HVG Sokolow (mm) }\end{array}$ & $\begin{array}{r}66.0 \\
382.7 \\
31.0\end{array}$ & $\begin{array}{r}65.8 \\
387.9 \\
30.5\end{array}$ & $\begin{array}{l}>0.05 \\
>0.05 \\
>0.05\end{array}$ \\
\hline \multicolumn{4}{|l|}{ Appearance-distribution of point $\mathrm{J}$} \\
\hline $\begin{array}{l}\text { Inferolateral slurring (D2D3Vf-V5V6) } \\
\text { Lateral slurring (DlaVI-V5V6) } \\
\text { Lower slurring (D2D3Vf) } \\
\text { Inferolateral notching (D2D3Vf-V5V6) } \\
\text { Lateral notching (DlaVI-V5V6) } \\
\text { Lower notching (D2D3Vf) }\end{array}$ & $\begin{aligned} 18 & (37.8 \%) \\
7 & (14.5 \%) \\
6 & (12.5 \%) \\
8 & (16.6 \%) \\
5 & (10.5 \%) \\
4 & (8.3 \%)\end{aligned}$ & $\begin{aligned} & 19(39.5 \%) \\
& 7(14.5 \%) \\
& 6(12.5 \%) \\
& 8(16.6 \%) \\
& \quad 4(8.3 \%) \\
& 4(8.3 \%)\end{aligned}$ & $\begin{array}{l}>0.05 \\
>0.05 \\
>0.05 \\
>0.05 \\
>0.05 \\
>0.05\end{array}$ \\
\hline \multicolumn{4}{|l|}{ Appearance of ST segment elevation } \\
\hline $\begin{array}{l}\text { Ascending ST elevation } \\
\text { Horizontal or descending ST elevation }\end{array}$ & $\begin{array}{l}29(60.4 \%) \\
19(39.6 \%)\end{array}$ & $\begin{array}{l}28(58.4 \%) \\
20(41.6 \%)\end{array}$ & $\begin{array}{l}>0.05 \\
>0.05\end{array}$ \\
\hline
\end{tabular}

\section{DISCUSSION:}

\section{Prevalence of ERP}

In our selected healthy population, we found an ERP prevalence of $19.2 \%$, while this has been estimated between $3.3 \%$ and $13.1 \%$ in the general population. In 2008, Haissaguere determined a prevalence of $5 \%$ in a control population of 412 patients whose mean age was $36.5 \pm 12$ years $^{2}$. Tikkanen in a cohort of 10,864 patients found a prevalence of ERP of $5.8 \%(7.1 \%$ in men and $4.6 \%$ in women) with a mean age of $44 \pm 8.5$ years. $^{3}$ The MONICA/KORA study found a prevalence of $13.1 \%$ in a population of 6213 patients with a mean age of $52 \pm 10$ years. ${ }^{4}$ In 2011, Noseworthyl also showed in the study of two large American 
and European cohorts comprising 3995 and 5489 patients, respectively, an ERP prevalence of $4.5 \%$ across both populations. The prevalence was $6.1 \%$ in the U.S. population and $3.3 \%$ in the European population. ${ }^{5}$ Our young and athletic population therefore does not strictly represent the general population described in the literature. Nor can we compare with a population of elite athletes as studies on this population show a higher prevalence of ERP at $20 \%$. Noseworthy, in a study including 879 athletes, showed an ERP prevalence of $25.1 \%$ (male athletes: $33 \%$ female athletes: $11 \%) .{ }^{10}$ Tikkanen conducted a study involving 62 Finnish athletes aged 13-15 years and 503 Americans aged 17-24 years. $^{7}$ Among Finnish athletes, the prevalence of ERP was $44 \%$, while it was estimated at $30 \%$ among Americans. These different clinical studies on the general population and athletes show that the presence of ERP is influenced by genetic and clinical factors. Thus, the male predominance and geographical distribution of ERP would support a genetic contribution. ${ }^{11}$ Age, physical activity, and vagal tone are the factors influencing the presence of an ERP with a decline in prevalence as the patient's age increases. In practice, this translates into a more common ERP in athletic males whose ECG is characterized by a lower HR and a shorter QT interval than subjects without ERP. This translates in our population as an increased prevalence of ERP.

\section{Persistence of ERP}

Elevation of the J point defining the appearance of ERP is dynamic and variable over time. ${ }^{2}$ An elevated J point was therefore shown shortly before the occurrence of an electrical storm in patients with an implantable cardioverter defibrillator (ICD) as secondary prevention of SD in a healthy heart. ${ }^{12}$ The dynamic nature of ERP was also demonstrated by Noseworthy in a healthy population of elite athletes. His study showed an increased prevalence of ERP (37.2\%) in a population of 146 male athletes (soccer and rowing) and a significant increase in this $(52.7 \%$, $\mathrm{P}=0.003$ ) after a training period of 90 days $\left(\approx 20\right.$ hours/week). ${ }^{10}$ In the general population, ERP is variable over time and regresses with age. Indeed, Stein and Adhikarla showed a regression in ERP in more than $50 \%$ of cases in 244 patients (mean age $42 \pm 13$ years) from a cohort of 29,281 subjects after 10 years of follow-up. ${ }^{13,14}$ In our study, ERP persisted in $97.9 \%$ of patients without any change in the appearance, amplitude, and distribution of point $\mathrm{J}$. These differing results in the literature can be explained by the lack of influence of aging in our population over 5 years. They ultimately remained young $(<40$ years) and statistically identical at the two periods (sport and HR).

\section{ERP Prognosis after 5 Years}

Over the period of 5 years, no clinical event such as death, syncope, or SD occurred. Clinically, our population at baseline had no clinical risk criteria of SD. The most common ECG appearance of repolarization was a slurring type point J elevation $<2 \mathrm{~mm}(64.5 \%)$ in the lower, lateral, and inferolateral leads, associated with an ascending ST segment elevation. This appearance is suggests a mild form. Indeed, Tykkanen showed that patients with a point $\mathrm{J}$ elevation higher than $2 \mathrm{~mm}$ have three times the risk of death from arrhythmias compared to a control population. ${ }^{3}$ The notching appearance of the point $\mathrm{J}$ in the lower and inferolateral branches is more often present in patients with a VF. ${ }^{8}$ Finally, RP+ patients associated with a horizontal or descending ST segment elevation had a poor prognosis. ${ }^{7}$

\section{Implication in Medical Expertise}

The discovery of an ERP in our military population requires us to have a pragmatic approach in medical prevention and expertise. Screening patients at risk of SD associated with ERP is achieved through a specific examination and a rigorous interpretation of the ECG, in particular investigating the previously described malignancy criteria. Upon the discovery of an ERP in an asymptomatic military officer and in the absence of malignancy criteria, there will be no restriction in terms of fitness to work or physical activity. In the presence of SD in the family and/or unexplained syncope, officers undergo further investigations in a military hospital cardiology service (pharmacological tests, programmed ventricular stimulation). The symptomatic RP+ military officer may be limited in his professional practice if it presents specific risks (pilot, paratrooper, commando). The symptomatic $\mathrm{RP}+$ military officer in secondary prevention of SD has an ICD, but can no longer carry out his professional activity. ${ }^{12}$ 


\section{Study Limitations}

This is a single-center study of a specific population. The findings may not fully apply to the general population. The design of our study does not provide the continuous appearance of repolarization because it involves two ECG recordings carried out 5 years apart (ECG P1 and $\mathrm{P} 2$ ). The information is therefore incomplete. We provided an observational ECG study of ERP in military recruits, which lacks prognostic value and long-term follow-up data. The surface and implantable Holters allow continuous analysis thereof as well as specific information, in particular vagal tone information.

ERP is commonly found in young, athletic military officers, free of heart disease and cardiovascular risk factors. This ERP persists over 5 years and has a good prognosis. In our professional practice, the different periodic medical visits carried out on the military officers include evaluation of the risk of SD associated with ERP by examination and regular ECGs.

Acknowledgments: This study was approved by the army medical service ethics committee.

\section{REFERENCES}

1. . Massoure PL, Sacher F, Rigollaud JM, et al. Sudden cardiac death in young adult associated with early repolarization: Which consequences in military medicine? Medecine et Armées 2010;38:257-261.

2. Haïssaguerre M, Derval N, Sacher F, et al. Sudden cardiac arrest associated with early repolarization. N Engl J Med 2008;358:2016-2023.
3. Tikkanen JT, Anttonen O, Junttila MJ, et al. Long term outcome associated with Early repolarization on electrocardiography. N Engl J Med 2009;361:2529-2537.

4. Sinner MF, Reinhard W, Müller M, et al. Association of early repolarization pattern on ECG with risk of cardiac and all-cause mortality: A population-based prospective cohort study (MONICA/KORA). PLoS Med 2010;7:e1000314.

5. Noseworthy A, Tikkanen JT, Porthan $\mathrm{K}$, et al. The early repolarization pattern in the general population. J Am Coll Cardiol 2011;57:2284-2289.

6. Abe A, Yoshino $\mathrm{H}$, Ishiguro $\mathrm{H}$, et al. Prevalence of J waves in 12- lead electrocardiogram in patients with syncope and no organic disorder. J Cardiovasc Electrophysiol 2007;18:S88.

7. Tikkanen J, Juntilla MJ, Anttonen O, et al. Early repolarization: Electrocardiographic phenotypes associated with favorable long term outcome. Circulation 2011;123:26662673.

8. Merchant FM, Noseworthy PA, Weiner RB, et al. Ability of terminal QRS notching to distinguish benign from malignant electrocardiographic forms of early repolarization. Am J Cardiol 2009;104:1402-1406.

9. Vinsonneau U, Pinon B, Paleiron $N$, et al. Prevalence of early repolarization patterns in a French military population at low cardiovascular risk: Implications for preventive medicine. Ann Noninvasive Electrocardiol 2013;18:436440.

10. Noseworthy PA, Weiner R, Kim J, et al. Early repolarization pattern in competitive athlete. Clinical correlates and the effect of exercise training. Circ Arrhythm Electrophysiol 2011;4:432-440.

11. Nam GB, Ko KH, Kim J, et al. Mode of onset of ventricular fibrillation in patients with early repolarization pattern vs. Brugada syndrome. Eur Heart J 2010;31:330-339.

12. Haissaguere $M$, Sacher $F$, Nogami A, et al. Characteristics of recurrent ventricular fibrillation associated with inferolateral early repolarization role of drug therapy. J Am Coll Cardiol 2009;53:612-619.

13. Adhikarla C, Boga M, Wood AD, Froelicher VF. Natural history of the electrocardiographic pattern of early repolarization in ambulatory patients. Am J Cardio 2011;108: 1831-5.

14. Stein $R$, Sallam $K$, Adhikarla $C$, et al. Natural history of early repolarization in the inferior leads. Ann Noninvasive Electrocardiol 2012;17:331-339. 